\title{
Polymorphisms but Not Mutations of the KCNQ1 Gene Are Associated with Lone Atrial Fibrillation in the Chinese Han Population
}

\author{
Hui-min Chu, ${ }^{1}$ Ming-jun Feng, ${ }^{1}$ Yi-gang Li, ${ }^{2}$ Yi-xin Zhang, ${ }^{3}$ Ji-fang Ma, \\ Bin He, ${ }^{1}$ Yi-bo Yu, ${ }^{1}$ Jing Liu, ${ }^{1}$ and Xiao-min Chen ${ }^{1}$ \\ ${ }^{1}$ Department of Cardiology, Ningbo No. 1 Hospital Affiliated to Medical College of Ningbo University, \\ 59 Liuting Street, Haishu District, Ningbo 315211, China \\ ${ }^{2}$ Department of Cardiology, Xinhua Hospital Affiliated to Shanghai Jiaotong University School of Medicine, \\ 227 Chongqing Southern Road, Shanghai 200092, China \\ ${ }^{3}$ Department of Human Population Genetics, Institute of Molecular Medicine, Peking University, \\ 5 Yiheyuan Road, Beijing 100871, China \\ Correspondence should be addressed to Xiao-min Chen; markchu@126.com
}

Received 10 January 2013; Accepted 18 March 2013

Academic Editors: Y. Du and Y. Wang

Copyright (C) 2013 Hui-min Chu et al. This is an open access article distributed under the Creative Commons Attribution License, which permits unrestricted use, distribution, and reproduction in any medium, provided the original work is properly cited.

Background. Recent studies suggest that mutation of the slow delayed rectifier potassium channel (IKs) contributes to familial atrial fibrillation (FAF). In the current study, we identified common genetic variants of KCNQ1 and explored the potential association between KCNQ1 polymorphism with lone AF (LAF). Methods. Clinical data and blood samples were collected from 190 Han Chinese patients with sporadic AF and matched healthy controls. Variants of the KCNQ1 gene were identified using single-strand conformational polymorphism (SSCP) analysis. A case-control association study in KCNQ1 identified six known single-nucleotide polymorphisms (SNPs) during SSCP screening of the 190 LAF patients and 190 healthy controls. Results. One of the SNPs in KCNQ1 was strongly associated with LAF; significant allelic association was detected rs59233444 $(P=0.013, \mathrm{OR}=1.469,95 \%$ confidence interval (CI): 1.083-1.993). A multiple regression analysis indicated that rs59233444 is an independent risk factor for LAF. Twelve new variants were identified in KCNQ1, including one in the $5^{\prime}$-UTR, two in the $3^{\prime}$-UTR, six in introns, two synonymous substitutions, and one missense substitution. Variants c.1009C $>$ T, c.1860C $>$ T, and c. $+2285 \mathrm{C}>\mathrm{T}$ were not present in the 190 controls, and the others were identified in controls at various frequencies. Conclusions. rs59233444, a common SNP but not mutation in the coding regions of the KCNQ1 gene, is a risk factor for LAF in Chinese Han population.

\section{Background}

Atrial fibrillation (AF), a common type of cardiac arrhythmia, is a major risk factor for stroke, heart failure, and other cardiovascular morbidities $[1,2]$. In $15 \%-30 \%$ of the $\mathrm{AF}$ patients, no underlying heart disease could be identified; these cases are referred to as lone $\mathrm{AF}(\mathrm{LAF})$. In about $5 \%$ of the cases, family history could be clearly established; these are known as familial AF (FAF). Genetic defect was first reported in Chinese kindred in 2003 [3]. A mutation (S140G) was found in the first transmembrane spanning domain of the cardiac slow delayed rectifier potassium channel (IKs), encoded by the KCNQ1 gene [4]. Functional analysis of the mutation revealed a "gain-of-function" effect on both KCNQ1-KCNE1 and KCNQ1-KCNE2 ion channels. Since then, "gain-of-function" mutations in other genes encoding potassium ion channels have been found to be associated with FAF, including KCNE2 [5], KCNE3 [6], KCNA5 [7], and KCNJ2 [8]. Q147R [9], R231C [10], and S209P [11] mutations of the KCNQ1 gene were also reported.

Interestingly, the gain-of-function mutation of KCNE5 is also linked to LAF [12]. The KCNE5 gene product MiRP4 suppresses the IKs current and downregulates the $\beta$-subunit of the KCNQ1. The mutant KCNE5 L65F fails to suppress 
IKs, yielding a current indistinguishable from that recorded in the absence of KCNE5. There is evidence of a heritable contribution to LAF, where a polymorphism (S38G) in Mink (KCNE1) was associated with nonfamilial AF [13] (the sporadic LAF). These results indicate that a polymorphism (S38G) and a mutation (S140G) in different genes (KCNE1 and KCNQ1, resp.) encoding different subunits of the same ionic channel (IKs) may be responsible for the development of nonfamilial and familial AF. These results also suggest that familial and nonfamilial AF (sporadic LAF) may share a common pathological mechanism and provide justification to test KCNQ1 as a candidate gene for LAF.

In the current study, we compared KCNQ1 variants in 190 Chinese Han patients with LAF and 190 healthy controls. We also performed a case-control association study for several common SNPs in KCNQ1.

\section{Methods}

2.1. Study Subjects. Consecutive patients with LAF referred to the cardiology department of Ningbo No. 1 Hospital and Shanghai Xinhua Hospital from June 1, 2007 to September 27, 2009 were enrolled. AF was defined as replacement of the sinus $\mathrm{P}$ waves by rapid oscillations of fibrillation waves that varied in size, shape, and timing and were associated with an irregular ventricular response when atrioventricular conduction was intact. LAF was defined as AF occurring in patients $<60$ years of age without identifiable causes, including hypertension, overt structural heart disease, or thyroid dysfunction. FAF was defined as the presence of LAF in one or more first-degree relative of the indexed case. Each patient underwent a physical examination and a standardized interview to identify past medical conditions, medications, symptoms, family history, and possible triggers for the initiation of AF. All patients were evaluated by 12-lead electrocardiogram (ECG), echocardiogram, and laboratory studies.

Normal control individuals were selected from a crosssectional, population-based cohort of 190 individuals from Chinese Han people in Southern China. Each subject underwent a comprehensive medical evaluation consisting of a medical history, a physical examination, echocardiography, and electrocardiography. We selected age-, gender-, and ethnicity-matched controls for our study from this population cohort. Control subjects did not have a history of or clinical evidence for AF or any structural disease. Genomic DNA was isolated from peripheral blood leukocytes using standard protocols with the Wizard Genomic DNA Purification Kit (Agilent). This study was approved by the Institution of Ningbo Medical Societies, and all patients gave written informed consent.

2.2. Mutation Analysis by SSCP and DNA Sequencing. Exons and exon-intron boundaries of the KCNQ1 gene were amplified by PCR using standard conditions with primers designed from the published KCNQ1 sequences in the NCBI database (Accession number: NG_008935.1). PCR was performed in a $25 \mu \mathrm{L}$ volume containing $200 \mathrm{pmol}$ of each primer, $10 \mathrm{ng}$ of genomic DNA, $2.5 \mu \mathrm{L}$ of $10 \times$ PCR buffer with $1.5 \mathrm{mmol}$ $\mathrm{MgCl}_{2}, 100 \mu \mathrm{mol}$ deoxynucleotide triphosphates, and 1 unit of Taq DNA polymerase (Solarbio).

Amplified samples were diluted twofold with $6 \mu \mathrm{L}$ of formamide buffer (90\% formamide, $1 \mathrm{mmol}$ EDTA, $0.2 \%$ bromophenol blue, and $0.1 \%$ xylene cyanol). The mixture was denatured at $96^{\circ} \mathrm{C}$ for 3 minutes, then cooled rapidly on ice, and held for 5 minutes. For each sample, $7 \mu \mathrm{L}$ was loaded onto $10 \%$ nondenaturing polyacrylamide gels (acrylamide to bisacrylamide ratio $=40: 10$ ) and electrophoresed at $80 \mathrm{~V}$ for one half hour to two hours at room temperature. The gel was stained with $0.1 \%$ silver nitrate and visualized with a $2 \%$ $\mathrm{NaOH}$ solution (containing $0.1 \%$ formaldehyde). Aberrant conformers were directly sequenced with ABI 3130XL instruments (Applied Biosystem), and the sequence was analyzed with Sequence Scanner Software (Version 1.0).

2.3. SNP Genotyping. Subsequently, a case-control association study was performed with known SNPs, which were identified from mutation screening. Six SNPs (rs59233444, rs1057128, rs163150, rs760419, rs163160, and rs2075870) were genotyped using direct DNA sequencing (ABI 3130XL, Applied Biosystems). The PCR products were sequenced using forward and/or reverse PCR primers.

2.4. Statistical Analysis. Hardy-Weinberg equilibrium calculations were applied to analyze the distribution of genotypes. A $\chi^{2}$ test was used to compare allele and genotype frequencies between the cases and controls and to obtain odds ratios (ORs) with 95\% confidence intervals (CIs). The SPSS statistical software (Version 18.0) was used for analyzing LD, and haplotypes were calculated using the Haploview software package. Statistical differences were judged significant at $P<$ 0.05 . The multivariate logistic analysis included age, gender, diabetes, drinking, and smoking habits as covariates.

\section{Results}

3.1. Characteristics of the Study Population. A total of 190 patients with LAF and 190 controls were enrolled for the study. Among the 190 patients with LAF, 3 had at least one first-degree relative with AF. The clinical characteristics of these 190 patients are summarized in Table 1. No significant differences were seen between the case patients and control subjects with regard to age, sex, diabetes, smoking and drinking habits, left ventricle ejection fraction, left ventricular enddiastolic diameter, and left ventricular end-systolic diameter. However, the left atrial dimension in the case patient cohort is larger than that in the control cohort $(38.8 \pm 6.6 \mathrm{~mm}$ versus $35.4 \pm 4.8, P<0.01)$. All subjects in our study were of Han ethnic origin.

3.2. Identification of Variants in LAF. To identify mutations or rare polymorphisms associated with $\mathrm{AF}$, all exons and exon-intron boundaries of KCNQ1 were screened by SSCP analysis. PCR products of aberrant conformers were directly sequenced to identify polymorphisms. A representative portion of the aberrant conformers found in LAF by SSCP 
TABLE 1: Clinical characteristics of the study population.

\begin{tabular}{lccc}
\hline & $\begin{array}{c}\text { Case } \\
(n=190)\end{array}$ & $\begin{array}{c}\text { Control } \\
(n=190)\end{array}$ & $P$ \\
\hline Age $^{\mathrm{a}}$ (years, mean \pm SD) & $55.4 \pm 6.3$ & $55.2 \pm 7.6$ & 0.211 \\
Gender (female, $n(\%))^{68(35.8 \%)}$ & $71(37.4)$ & 0.831 \\
Diabetes $^{\mathrm{b}}(n(\%))$ & $8(4.2 \%)$ & $10(5.3 \%)$ & 0.810 \\
Smoking habit $(n(\%))$ & $60(31.6 \%)$ & $54(28.4 \%)$ & 0.576 \\
Drinking habit $(n(\%))$ & $55(33.7 \%)$ & $43(22.6 \%)$ & 0.197 \\
Family history & $3(1.6 \%)$ & $\mathrm{NA}$ & $\mathrm{NA}$ \\
Left atrial dimension $(\mathrm{mm})$ & $38.8 \pm 6.6$ & $35.4 \pm 4.8$ & $<0.01$ \\
Left ventricle ejection & $64.8 \pm 8.9$ & $66.1 \pm 9.1$ & 0.215 \\
fraction $(\%)$ & $48.9 \pm 4.9$ & $48.9 \pm 7.2$ & 0.542 \\
LVEDD $(\mathrm{mm})$ & $31.3 \pm 4.2$ & $31.1 \pm 5.6$ & 0.253 \\
\hline LVESD $(\mathrm{mm})$ & & &
\end{tabular}

NA: data not available.

${ }^{\mathrm{a}}$ Age was defined as the age at the sample collection.

${ }^{b}$ Diabetes was defined as ongoing therapy of diabetes or a fasting plasma glucose level of $\geq 7.0 \mathrm{mmol}$.

and DNA sequencing is shown in Figure 1. A total of 12 variants were identified by our analysis (Table 2). One variant at the $5 /$-UTR, c. $-22 \mathrm{~T}>\mathrm{C}$, was detected in 2 patients and 1 control, indicating that this variant is a rare polymorphism. Six variants were found in the exon-intron boundaries, including c.511-19_511 18delTG, c.1128+3G $>$ A, c.1590+31A $>$ T, c. $1684+23 \mathrm{G}>\mathrm{A}, \mathrm{c} .1685-43 \mathrm{G}>\mathrm{A}$, and c.1795-18C $>\mathrm{T}$. We analyzed these six intronic variants with Human Splicing Finder (version 2.4.1) and found that they were unlikely to affect a splicing site. Three variants were identified in the coding region of KCNQ1, including two synonymous mutations, c. $1009 \mathrm{C}>\mathrm{T}$ and c. $1860 \mathrm{C}>\mathrm{T}$, that were only detected in three total patients and were not found in the controls, suggesting that they are potentially mutations. The other missense change was identified in exon16, c.1945G $>A$, and results in a $\mathrm{D}$ to $\mathrm{N}$ substitution at amino acid 649. This appears to be a polymorphism that is not associated with LAF, because it was present in four of the cases and five of the controls. Two variants were identified in the 3/-UTR, one of which, c. $+2285 \mathrm{C}>\mathrm{T}$, was found in one patient out of 190 cases and in none of the 190 controls. The other variant, c. $+2976 \mathrm{G}>\mathrm{A}$, was found in five of 190 LAF patients and in three of the 190 controls. We analyzed these two variants with Patrocles Finder (http://www.patrocles.org/) and did not find evidence of microRNA binding; therefore, they are likely rare nonfunctional polymorphisms. In addition to the 12 novel variants that we reported above, we found 12 known polymorphisms with different frequencies in the cohort of 190 LAF patients (Table 3).

3.3. Association of SNPs in KCNQ1 with LAF. Mutation analysis of all translated KCNQ1 exons in 190 LAF patients did not reveal a single mutation that clearly altered the splice junction or changed amino acid polarity. Therefore, we focused on six previously characterized SNPs that we identified in our cohort from gene mutation sequencing. Genotyping for six SNPs was done through direct DNA sequencing of rs59233444, rs1057128, rs163150, rs760419, rs163160, and rs2075870. The genotypic frequencies of the six SNPs in the controls were not significantly different from Hardy-Weinberg equilibrium. One of the six SNPs was associated with LAF in the additive and dominant genetic patterns (Table 4). For rs59233444, the genotype distribution is significantly different between (-/-, GG-, GG/GG) LAF patients and controls $(49.5,44.2$, and $6.3 \%$ versus $34.7,56.8$, and $8.5 \%, P=0.014)$. An allelic association with LAF was found by both [2] analysis and a regression test (Table 5). The GG minor allele frequency was $36.8 \%$ in the LAF group, compared with $28.4 \%$ in the normal controls (OR 1.469, $95 \%$ CI: 1.083-1.993, $P=0.013$ ), and the A minor allele frequency was $23.4 \%$ in the LAF patients, compared with $16.3 \%$ in the normal controls (OR 1.885, 95\% CI: 1.328-2.676, $P<0.01$ ) (Table 6).

In this study, we found rs59233444 were the risk factors for LAF. Moreover, we analyzed all confounding factors for LAF including sex, age, smoking, drinking and hypertension using multiple regression analysis. We found that rs59233444 was the independent risk factor for LAF excluding other risk factors (Table 7). We performed a linkage disequilibrium test and demonstrated a low $\mathrm{LD}$ for rs59233444 $\left(\mathrm{D}^{\prime}=0.342\right)$ (data not shown).

\section{Discussion}

KCNQ1 (KVLOT1) channel subunits coassemble with KCNE1 (Mink) subunits to form channels that conduct the slow delayed rectifier $\mathrm{K}^{+}$current, $I K_{s}$ in the heart which is important for normal termination of the plateau phase, and repolarization of atrial and ventricular action potentials. Mutations in KCNQ1 were first identified as being the molecular basis of autosomal dominant atrial fibrillation in a single family from China in 2003 [3]. Subsequent studies have implicated potassium ion channel mutations in the pathogenesis of AF $[3,5-8,10-12]$. Although the study of rare familial forms of atrial fibrillation provides insight into the molecular pathways involved in selective cases of the disease, these genetic defects may not be representative of the pathogenesis in the more common, nonfamilial forms found in sporadic AF patients. Therefore, we focused on whether the KCNQ1 gene was associated with LAF and screened for KCNQ1 mutations in a cohort of 190 unrelated individuals with LAF.

Despite a plausible rationale for $K C N Q 1$ as a candidate gene for LAF, we did not identify any KCNQ1 mutations in our cohort of 190 patients with sporadic LAF. There are several possible explanations for this. First, although AF has an inheritance tendency recognized by many studies, most of the gene mutations found in AF are those that are associated with a family history $[3,6-8,10-12,14-18]$. Patients with sporadic AF are more likely to be associated with functional polymorphisms rather than mutations [19-23]. Second, AF is a genetically heterogeneous disorder, so a large sample is generally required to sufficiently screen for KCNQ1 mutations that cause LAF. Finally, certain types of KCNQ1 mutations would be missed by our SSCP methodology owing to this 
TABLE 2: List of all KCNQ1 variants identified in the present study.

\begin{tabular}{|c|c|c|c|c|}
\hline Variation & Amino acid change & Frequency in patients & Conserved & Frequency in controls \\
\hline c. $-22 \mathrm{~T}>\mathrm{C}$ & - & $2 / 190$ & No & $1 / 190$ \\
\hline c.51119_51118delTG & - & $2 / 190$ & No & $3 / 190$ \\
\hline c. $1009 \mathrm{C}>\mathrm{T}$ & A336A & $1 / 190$ & Yes & $0 / 190$ \\
\hline c. $1128+3 \mathrm{G}>\mathrm{A}$ & - & $2 / 190$ & No & $1 / 190$ \\
\hline c. $1590+31 \mathrm{~A}>\mathrm{T}$ & - & $5 / 190$ & No & $3 / 190$ \\
\hline c. $1684+23 \mathrm{G}>\mathrm{A}$ & - & $2 / 190$ & No & $3 / 190$ \\
\hline c. $1685-43 \mathrm{G}>\mathrm{A}$ & - & $1 / 190$ & No & $2 / 190$ \\
\hline c. $1795-18 \mathrm{C}>\mathrm{T}$ & - & $3 / 190$ & No & $2 / 190$ \\
\hline c. $1860 \mathrm{C}>\mathrm{T}$ & $\mathrm{H} 620 \mathrm{H}$ & $2 / 190$ & Yes & $0 / 190$ \\
\hline c. $1945 \mathrm{G}>\mathrm{A}$ & D649N & $4 / 190$ & No & $5 / 190$ \\
\hline c. $+2285 \mathrm{C}>\mathrm{T}$ & - & $1 / 190$ & Yes & $0 / 190$ \\
\hline c. $+2976 \mathrm{G}>\mathrm{A}$ & - & $5 / 190$ & No & $3 / 190$ \\
\hline
\end{tabular}
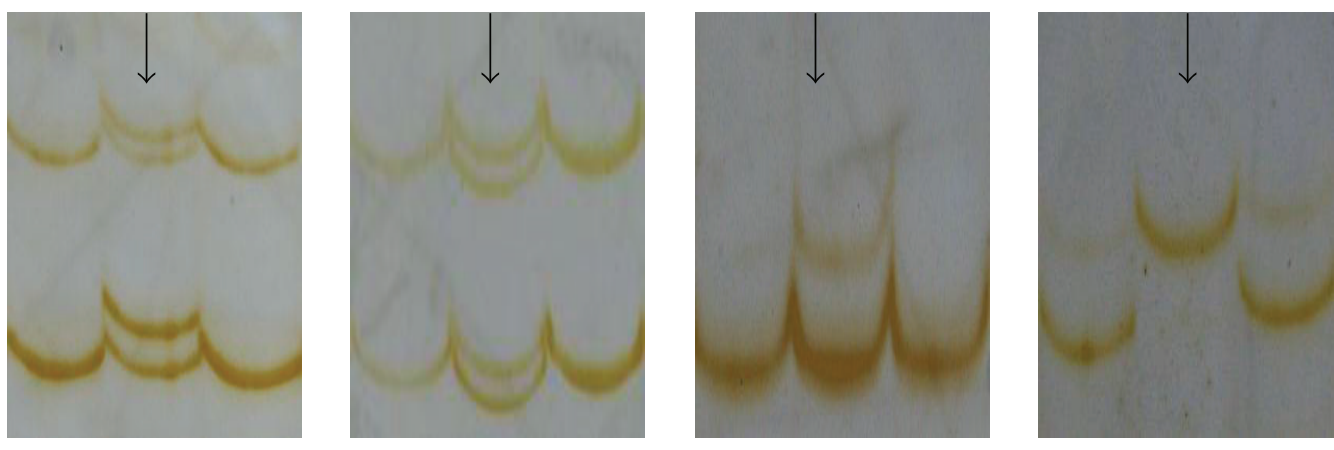

(a)

c. $-22 \mathrm{~T}>\mathrm{C}$
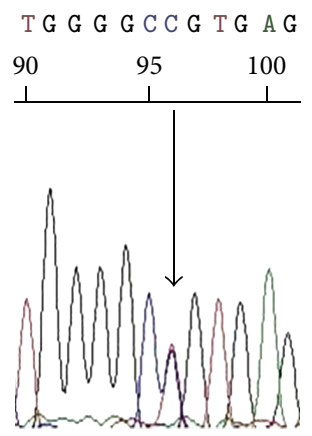

c. 511-19_511-18delTG

CAC A CTG TG T GGG G

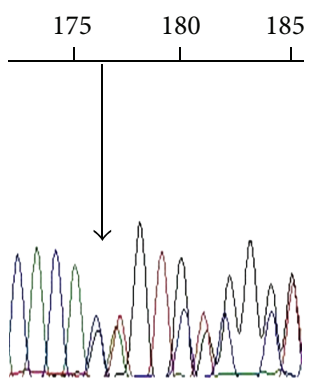

c. $1860 \mathrm{C}>\mathrm{T}$
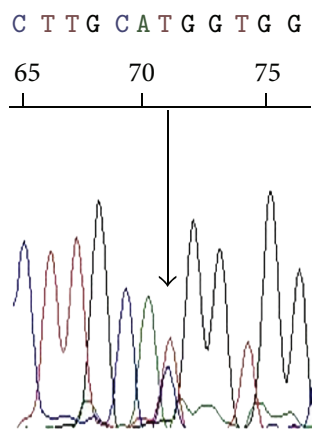

c. $+2976 \mathrm{G}>\mathrm{A}$

T G G G G C C G T G A G

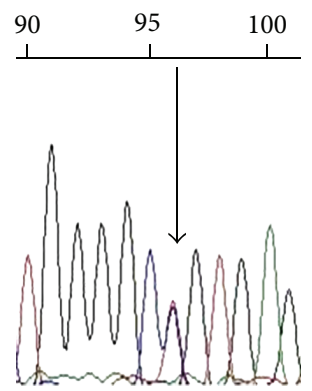

(b)

FIGURE 1: A representative image of an aberrant single-strand conformation found in the LAF using the single-strand conformation polymorphism (SSCP) procedure (a) and confirmed with direct DNA sequencing (b).

method's $<100 \%$ sensitivity. Despite these limitations, our results are consistent with other published results [24].

In addition to the 12 variants in KCNQ1 that we identified in LAF cases, we uncovered 12 known SNPs from LAF groups at different frequencies. After initial comparison of each of the 12 SNP frequencies that we obtained from the case group with the Han Chinese population in HapMap, six of twelve SNPs were targeted for the case-control association study. Finally, one of the six SNPs was determined to be associated with LAF which located in the KCNQ1 intron2. The GG minor allele conferred an odds ratio (OR) for developing LAF of 1.469 (95\% CI: $1.083-1.993, P=0.013$ ), and the A minor allele conferred a higher OR of 1.885 (95\% CI: 1.328-2.676, $P<0.01)$. After correcting for the confounding factors, rs59233444 was found to be a risk factor for LAF. Given the incidence of AF, it would be of importance to determine the functional relevance of the one SNP. It is formally possible that the association described here is attributable to LD 
TABLE 3: Twelve known polymorphisms found in LAF patients.

\begin{tabular}{lcccc}
\hline SNPs & Regions & $\begin{array}{c}\text { Minor } \\
\text { allele }\end{array}$ & $\begin{array}{c}\text { Amino acid } \\
\text { change }\end{array}$ & $\begin{array}{c}\text { Han Chinese } \\
\text { MAF }\end{array}$ \\
\hline rs1800170 & Exon2 & T & I145I & 0.091 \\
rs59233444 & Intron2 & GG & - & N.A \\
rs12786951 & Intron6 & G & - & 0.489 \\
rs12577654 & Intron12 & T & - & 0.057 \\
rs760419 & Intron12 & G & - & 0.318 \\
rs163160 & Intron12 & G & - & 0.244 \\
rs2075870 & Intron12 & A & - & 0.170 \\
rs1057128 & Exon13 & A & S546S & 0.289 \\
rs163150 & Intron14 & A & - & 0.289 \\
rs2519184 & $3^{\prime}$-UTR & A & - & 0.034 \\
rs45510192 & $3^{\prime}$-UTR & A & - & N.A \\
rs394656 & $3^{\prime}$-UTR & C & - & N.A \\
\hline
\end{tabular}

MAF (minor allele frequency) of Han Chinese is based on HapMap Data; N.A: not available from HapMap Data for Han Chinese population.

TABLE 4: Genotypic association of one SNP in KCNQ1 with LAF in the Han Chinese population.

\begin{tabular}{lccc}
\hline SNP & Model & $P$ & OR (95\% CI) \\
\hline \multirow{3}{*}{ rs59233444 } & Add & 0.014 & $1.579(1.128-2.210)$ \\
& Rec & 0.432 & $1.364(0.627-2.967)$ \\
& Dom & 0.004 & $1.840(1.218-2.779)$ \\
\hline
\end{tabular}

Add: additive model; Rec: recessive model; Dom: dominant model; $P: P$ value from logistic regression; OR (95\%): odds ratio with $95 \%$ confidence interval.

TABLE 5: Distribution of rs59233444 genotypes in patients and controls.

\begin{tabular}{lcccr}
\hline rs59233444 & $N$ & $-/-$ & $\begin{array}{c}\text { Genotype } \\
\text { GG/ }-\end{array}$ & GG/GG \\
\hline Case & 190 & $66(34.7 \%)$ & $108(56.8 \%)$ & $16(8.5 \%)$ \\
Control & 190 & $94(49.5 \%)$ & $84(44.2 \%)$ & $12(6.3 \%)$ \\
& \multicolumn{3}{c}{$\chi^{2}=8.471, P=0.014$} \\
\hline
\end{tabular}

TABLE 6: Allelic association for rs59233444 in LAF.

\begin{tabular}{lcccc}
\hline SNP & $\begin{array}{c}\text { Allele } \\
\text { (major/minor) }\end{array}$ & $\begin{array}{c}\text { MAF } \\
\text { (case/control) }\end{array}$ & $P$ & OR (95\% CI) \\
\hline Rs59233444 & - /GG & $0.368 / 0.284$ & 0.013 & $\begin{array}{c}1.469 \\
(1.083-1.993)\end{array}$ \\
\hline
\end{tabular}

between KCNQ1 gene polymorphisms or to another nearby susceptibility gene.

\section{Conclusions}

Mutations in the coding regions of KCNQ1 are not a common cause for LAF. Several SNPs were identified in coding and noncoding regions of KCNQ1. One SNP in KCNQ1 (rs59233444) is associated with LAF. Rs59233444 can be
TABLE 7: Multiple regression analysis for LAF in rs59233444.

\begin{tabular}{lc}
\hline Risk factors & $P$ value \\
\hline Sex & 0.789 \\
Age & 0.530 \\
Smoking & 0.689 \\
Drinking & 0.744 \\
Hypertension & $<0.001$ \\
rs59233444 & 0.005 \\
\hline
\end{tabular}

deemed as a risk factor for being susceptible to AF in Han Chinese people.

\section{Disclosure}

The authors declare that the submitted paper does not contain previously published material and is not under consideration for publication elsewhere. All authors listed have read the complete paper and have approved submission of the paper. The paper is truthful original work without fabrication, fraud, or plagiarism.

\section{Conflict of Interests}

The authors declare that they have no conflict of interests.

\section{Authors' Contribution}

Each author has made an important scientific contribution to the study and is thoroughly familiar with the primary data. Hui-min Chu and Ming-jun Feng are co-first authors, they contributed equally to this work; they carried out the molecular genetic studies, participated in the sequence alignment, and drafted the paper. Yi-xin Zhang participated in the sequence alignment. Yi-gang Li participated in the design of the study and performed the statistical analysis. Xiao-min Chen conceived of the study and participated in its design and coordination and helped to draft the paper. Bin $\mathrm{He}$ helped to revise the paper. Yi-bo Yu helped to collect the clinical data and the statistical analysis. Jing Liu participated in the design of the study. Ji-fang Ma provided the partial of case and control samples. All authors read and approved the final paper.

\section{Acknowledgments}

This work was funded by the City Natural Science Foundation of Ningbo, China (no. 2009A610176). The authors would like to thank Lin Zhao, Chong Wu (Department of Human Population Genetics, Institute of Molecular Medicine, Peking University), and Feng Jiang (Department of Cardiology, The Fourth Affiliated Hospital of Harbin Medical University).

\section{References}

[1] L. Frost, G. Engholm, S. Johnsen, H. Møller, and S. Husted, "Incident stroke after discharge from the hospital with a 
diagnosis of atrial fibrillation," American Journal of Medicine, vol. 108, no. 1, pp. 36-40, 2000.

[2] M. Grogan, H. C. Smith, B. J. Gersh, and D. L. Wood, "Left ventricular dysfunction due to atrial fibrillation in patients initially believed to have idiopathic dilated cardiomyopathy," American Journal of Cardiology, vol. 69, no. 19, pp. 1570-1573, 1992.

[3] Y. H. Chen, S. J. Xu, S. Bendahhou et al., "KCNQ1 gain-offunction mutation in familial atrial fibrillation," Science, vol. 299, no. 5604, pp. 251-254, 2003.

[4] Q. Wang, M. E. Curran, I. Splawski et al., "Positional cloning of a novel potassium channel gene: KVLQT1 mutations cause cardiac arrhythmias," Nature Genetics, vol. 12, no. 1, pp. 17-23, 1996.

[5] Y. Yang, M. Xia, Q. Jin et al., "Identification of a KCNE2 gainof-function mutation in patients with familial atrial fibrillation," American Journal of Human Genetics, vol. 75, no. 5, pp. 899-905, 2004.

[6] A. Lundby, L. S. Ravn, J. H. Svendsen, S. Haunsø, S. P. Olesen, and N. Schmitt, "KCNE3 mutation V17M identified in a patient with lone atrial fibrillation," Cellular Physiology and Biochemistry, vol. 21, no. 1-3, pp. 47-54, 2008.

[7] T. M. Olson, A. E. Alekseev, X. K. Liu et al., "Kv1.5 channelopathy due to KCNA5 loss-of-function mutation causes human atrial fibrillation," Human Molecular Genetics, vol. 15, no. 14, pp. 2185-2191, 2006.

[8] K. Calloe, L. S. Ravn, N. Schmitt et al., "Characterizations of a loss-of-function mutation in the Kir3.4 channel subunit," Biochemical and Biophysical Research Communications, vol. 364, no. 4, pp. 889-895, 2007.

[9] A. Lundby, L. S. Ravn, J. H. Svendsen, S. P. Olesen, and N. Schmitt, "KCNQ1 mutation Q147R is associated with atrial fibrillation and prolonged QT interval," Heart Rhythm, vol. 4, no. 12, pp. 1532-1541, 2007.

[10] D. C. Bartos, S. Duchatelet, D. E. Burgess et al., "R231C mutation in KCNQ1 causes long QT syndrome type 1 and familial atrial fibrillation," Heart Rhythm, vol. 8, no. 1, pp. 48-55, 2011.

[11] S. Das, S. Makino, Y. F. Melman et al., "Mutation in the S3 segment of KCNQ1 results in familial lone atrial fibrillation," Heart Rhythm, vol. 6, no. 8, pp. 1146-1153, 2009.

[12] L. S. Ravn, Y. Aizawa, G. D. Pollevick et al., "Gain of function in IKs secondary to a mutation in KCNE5 associated with atrial fibrillation," Heart Rhythm, vol. 5, no. 3, pp. 427-435, 2008.

[13] C. Fatini, E. Sticchi, M. Genuardi et al., "Analysis of minK and eNOS genes as candidate loci for predisposition to non-valvular atrial fibrillation," European Heart Journal, vol. 27, no. 14, pp. 1712-1718, 2006.

[14] R. L. Abraham, T. Yang, M. Blair, D. M. Roden, and D. Darbar, "Augmented potassium current is a shared phenotype for two genetic defects associated with familial atrial fibrillation," Journal of Molecular and Cellular Cardiology, vol. 48, no. 1, pp. 181-190, 2010.

[15] X. Ren, C. Xu, C. Zhan et al., "Identification of NPPA variants associated with atrial fibrillation in a Chinese GeneID population," Clinica Chimica Acta, vol. 411, no. 7-8, pp. 481-485, 2010.

[16] K. M. Brauch, L. Y. Chen, and T. M. Olson, "Comprehensive mutation scanning of LMNA in 268 patients with lone atrial fibrillation," American Journal of Cardiology, vol. 103, no. 10, pp. 1426-1428, 2009.

[17] D. M. Hodgson-Zingman, M. L. Karst, L. V. Zingman et al., "Atrial natriuretic peptide frameshift mutation in familial atrial fibrillation," New England Journal of Medicine, vol. 359, no. 2, pp. 158-165, 2008.

[18] T. Makiyama, M. Akao, S. Shizuta et al., "A novel SCN5A gainof-function mutation M1875T associated with familial atrial fibrillation," Journal of the American College of Cardiology, vol. 52, no. 16, pp. 1326-1334, 2008.

[19] L. Shi, C. Li, C. Wang et al., "Assessment of association of rs2200733 on chromosome 4q25 with atrial fibrillation and ischemic stroke in a Chinese Han population," Human Genetics, vol. 126, no. 6, pp. 843-849, 2009.

[20] P. T. Ellinor, K. L. Lunetta, N. L. Glazer et al., "Common variants in KCNN3 are associated with lone atrial fibrillation," Nature Genetics, vol. 42, no. 3, pp. 240-244, 2010.

[21] C. Zhang, G. H. Yuan, Z. F. Cheng, M. W. Xu, L. F. Hou, and F. P. Wei, "The single nucleotide polymorphisms of Kir3.4 gene and their correlation with lone paroxysmal atrial fibrillation in Chinese Han population," Heart Lung and Circulation, vol. 18, no. 4, pp. 257-261, 2009.

[22] Z. Zeng, C. Tan, S. Teng et al., "The single nucleotide polymorphisms of IKs potassium channel genes and their association with atrial fibrillation in a Chinese population," Cardiology, vol. 108, no. 2, pp. 97-103, 2007.

[23] L. Y. Chen, J. D. Ballew, K. J. Herron, R. J. Rodeheffer, and T. M. Olson, "A common polymorphism in SCN5A is associated with lone atrial fibrillation," Clinical Pharmacology and Therapeutics, vol. 81, no. 1, pp. 35-41, 2007.

[24] P. T. Ellinor, V. I. Petrov-Kondratov, E. Zakharova, E. G. Nam, and C. A. MacRae, "Potassium channel gene mutations rarely cause atrial fibrillation," BMC Medical Genetics, vol. 7, article 70, 2006. 

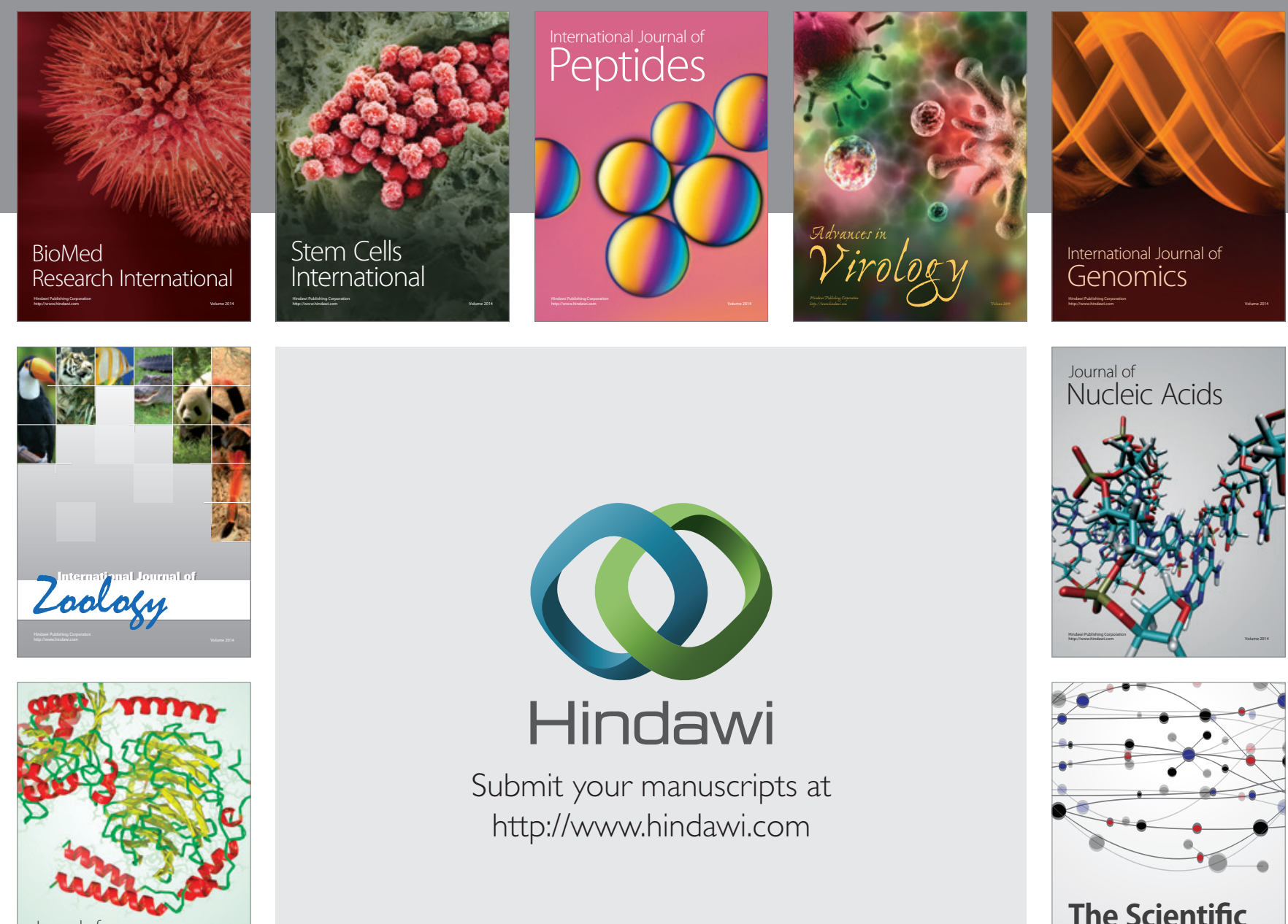

Submit your manuscripts at

http://www.hindawi.com

Journal of
Signal Transduction
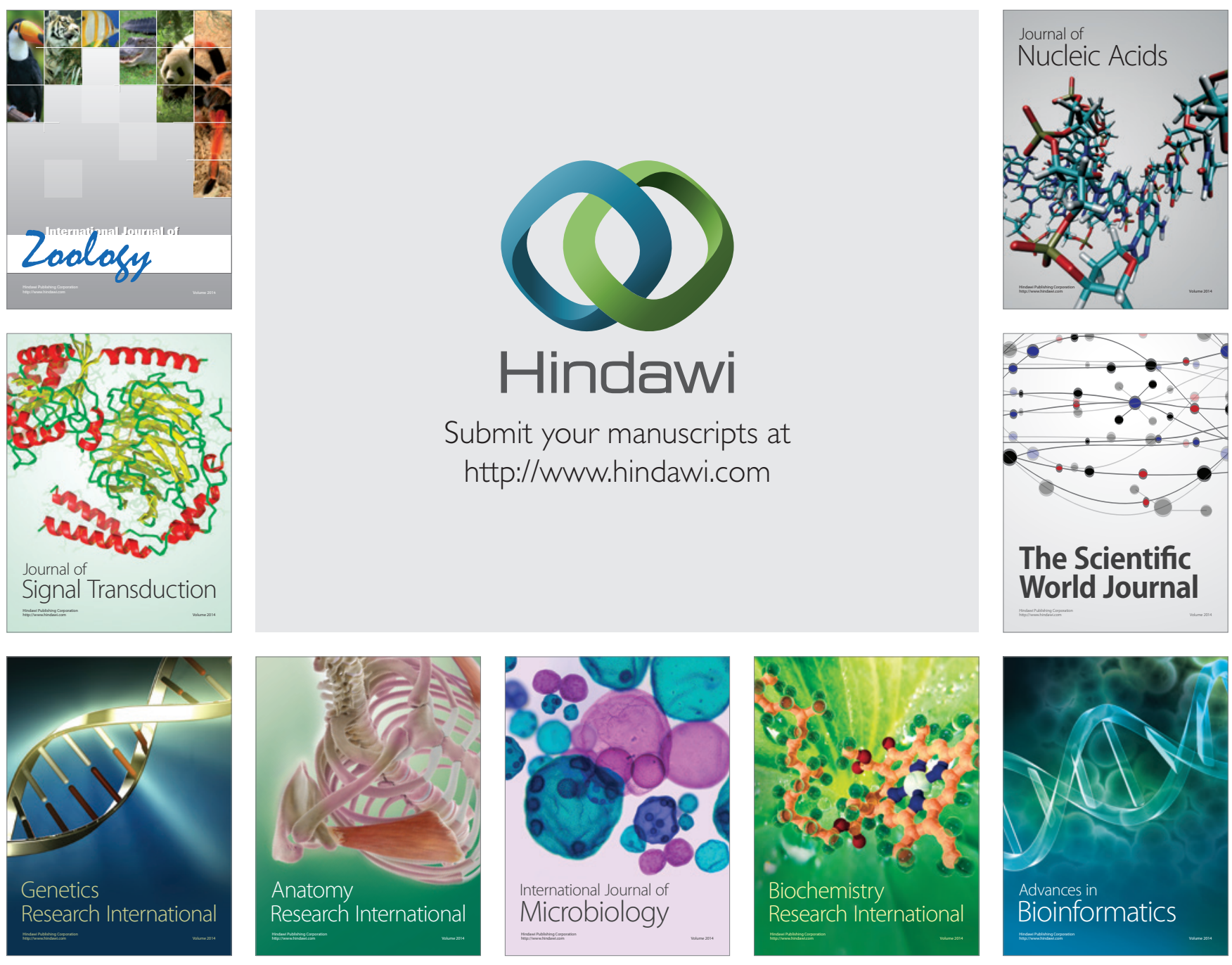

The Scientific World Journal
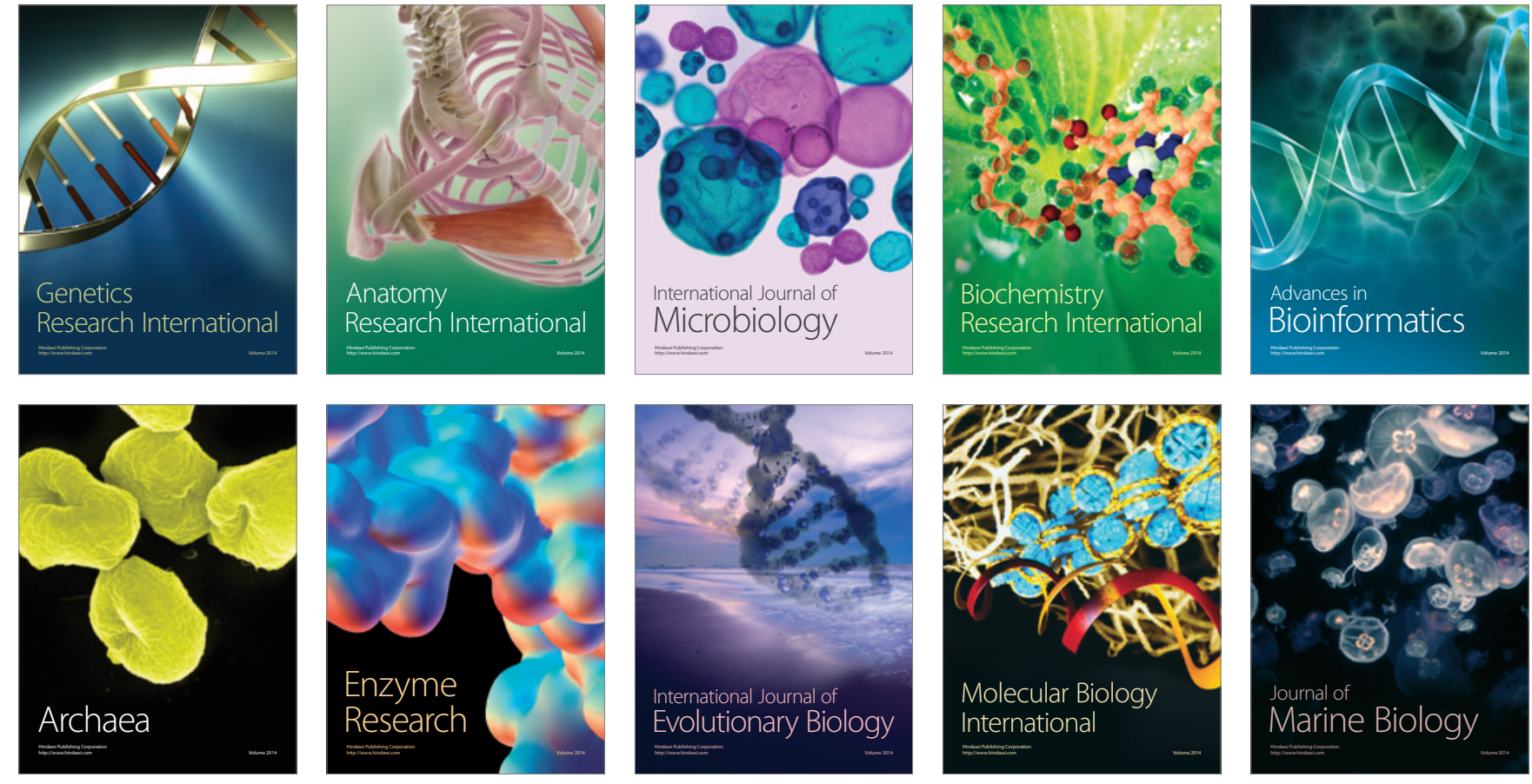